\title{
Chemotherapy for non-small cell lung cancer complicated by idiopathic interstitial pneumonia
}

\author{
TAKASHI KINOSHITA ${ }^{1}$, KOICHI AZUMA ${ }^{1}$, TETSURO SASADA ${ }^{2}$, MASAKI OKAMOTO $^{1}$, SATOSHI HATTORI ${ }^{3}$, \\ YOUHEI IMAMURA ${ }^{1}$, KAZUHIKO YAMADA ${ }^{1}$, MORIHIRO TAJIRI ${ }^{1}$, TSUKASA YOSHIDA $^{1}$, \\ YOSHIAKI ZAIZEN $^{1}$, AKIHIKO KAWAHARA ${ }^{4}$, KIMINORI FUJIMOTO $^{5}$ and TOMOAKI HOSHINO ${ }^{1}$ \\ ${ }^{1}$ Department of Internal Medicine, Division of Respirology, Neurology and Rheumatology; \\ ${ }^{2}$ Department of Immunology and Immunotherapy; ${ }^{3}$ Biostatistics Center, Kurume University; \\ Departments of ${ }^{4}$ Diagnostic Pathology and ${ }^{5}$ Radiology, Kurume University School of Medicine, Kurume, Japan
}

Received February 22, 2012; Accepted May 25, 2012

DOI: 10.3892/ol.2012.753

\begin{abstract}
Idiopathic interstitial pneumonia (IIP) is considered to be one of the risk factors for lung cancer (LC). However, therapeutic options for patients with LC complicated by IIP are not well established. In this study, we investigated the feasibility and efficacy of chemotherapy for patients with non-small cell lung cancer (NSCLC) complicated by IIP (NSCLC-IIP). We retrospectively analyzed 22 NSCLC-IIP patients who received chemotherapy. To determine how IIP affected the clinical outcomes in NSCLC, they were compared with 276 NSCLC patients without IIP, who were treated with chemotherapy alone. The response rate (partial response + stable disease) was $72.3 \%$ (17/22), whereas the incidence of acute exacerbation (AE) was $13.6 \%(3 / 22)$ in NSCLC-IIP patients treated with chemotherapy. NSCLC-IIP patients had significantly shorter survival compared with NSCLC patients without IIP $(\mathrm{P}<0.001)$ following chemotherapy, although the response rates to chemotherapy were not significantly different between the two groups. Multivariate analysis demonstrated that, in NSCLC patients receiving chemotherapy, IIP was a significantly unfavorable factor for progression-free and overall survival. Despite similar response rates to chemotherapy, NSCLC-IIP patients showed poorer prognosis than NSCLC patients without IIP, possibly due to the natural course of IIP Chemotherapy may be a feasible option for NSCLC-IIP, if the risks of adverse effects are acceptable.
\end{abstract}

Correspondence to: Dr Koichi Azuma, Department of Internal Medicine, Division of Respirology, Neurology and Rheumatology, Kurume University School of Medicine, 67 Asahi-machi, Kurume, Fukuoka 830-0011, Japan

E-mail: azuma@med.kurume-u.ac.jp

Key words: non-small cell lung cancer, idiopathic interstitial pneumonia, acute exacerbation, chemotherapy

\section{Introduction}

Lung cancer (LC) continues to be the leading cause of cancer mortality worldwide. Non-small cell lung cancer (NSCLC) is the most common type of LC, accounting for approximately $80 \%$ of all cases, and is classified into adenocarcinoma, squamous cell carcinoma and large cell carcinoma. Various therapeutic approaches have been developed and applied in accordance with the disease state of individual patients. Despite intensive studies of treatment modalities for NSCLC, the prognosis of affected patients remains poor (1).

Idiopathic interstitial pneumonia (IIP) is a group of slowly progressive pulmonary diseases which lead to respiratory insufficiency. IIP is a devastating parenchymal lung disease characterized by alveolar destruction, excess matrix production and varying levels of inflammation leading to impaired gas exchange. IIP has a poor prognosis, with a median survival time of 3 to 5 years from the time of diagnosis (2-4). The clinical course of IIP is usually chronic, but patients may occasionally experience episodes of acute respiratory worsening. Although these episodes may be secondary to special conditions, including pneumonia, pulmonary embolism, pneumothorax or cardiac failure, the term 'acute exacerbation (AE) of IIP' has been used when a cause for the acute respiratory worsening cannot be identified. AE of IIP is characterized by the acute or subacute onset of dyspnea with or without other symptoms, including cough and low-grade fever, and often progresses rapidly to respiratory failure requiring hospitalization and mechanical ventilation. Since no effective therapies are currently available, the prognosis of patients with $\mathrm{AE}$ of IIP remains extremely poor (2-4).

IIP has been considered to be one of the risk factors for LC. For example, Kawasaki et al reported that IIP was observed in $7.5 \%$ of surgically resected cases of LC (5). Although IIP patients show a higher incidence of $\mathrm{LC}$, with a relative risk of 7-14 (2-4,6,7), no standard therapy for LC complicated by IIP (NSCLC-IIP) has yet been established. In fact, patients with NSCLC-IIP are often followed up without standard treatments such as chemotherapy or radiotherapy, as there has been an underlying belief that chemotherapy may cause AE of IIP in 
NSCLC-IIP patients, although no concrete evidence has been reported. In clinical practice, NSCLC-IIP has been carefully treated with chemotherapy. Since there is limited information regarding the feasibility and efficacy of chemotherapy for NSCLC-IIP, in the present study we conducted a retrospective analysis of patients with NSCLC- IIP.

\section{Materials and methods}

NSCLC patients with or without IIP. We retrospectively examined LC patients with $(\mathrm{n}=57)$ and without $(\mathrm{n}=488)$ IIP between 1999 and 2008 at Kurume University Hospital (Kurume, Japan). This study was approved by the Institutional Review Board of Kurume University. To focus on the feasibility and efficacy of chemotherapy, we excluded NSCLC patients with or without IIP who had received treatments other than chemotherapy alone, including concurrent chemoradiotherapy, epidermal growth factor receptor-tyrosine kinase inhibitors (EGFR-TKIs) and surgery. Patients with an Eastern Cooperative Oncology Group (ECOG) performance status (PS) of 3 or 4, who were ineligible for chemotherapy, and those with small-cell lung cancers were also excluded. A full explanation of the potential risks and benefits was provided to 28 NSCLC-IIP patients; 22 received chemotherapy and the remaining 6 selected best supportive care (BSC). Tumors in the NSCLC-IIP patients receiving chemotherapy were diagnosed histologically as adenocarcinoma in 11 patients, squamous cell carcinoma in 7, large cell carcinoma in 2 and non-small cell carcinoma in 2 on the basis of the World Health Organization (WHO) criteria. The treatment regimens consisted of carboplatin (CBDCA) and paclitaxel (TXL; $\mathrm{n}=19)$, cisplatin-vinorelbine $(\mathrm{n}=2)$ and cisplatin-docetaxel $(n=1)$. As a control group, 276 NSCLC patients without IIP who received chemotherapy alone were examined. The tumor histology was as follows: 203 adenocarcinomas, 57 squamous cell carcinomas, 9 large cell carcinomas, 3 adenosquamous cell carcinomas and 4 non-small cell carcinomas (unclassified). The treatment regimens consisted of CBDCA-based $(n=192)$, cisplatin-based $(n=66)$ and non-platinum regimens [irinotecan + ifosfamide $(n=5)$, irinotecan + mitomycin $C$ $(n=1)$, gemcitabine + vinorelbine $(n=2)$, vinorelbine $(n=10)]$. The combination of CBDCA and TXL was the most frequently used regimen $(n=177)$. Details of the demographics, treatments and follow-up characteristics of the patients are shown in Table I. The patients were followed up until the time of mortality or September 2010. All underwent plain chest $\mathrm{X}$-ray examinations, computed tomography scans of the chest and upper abdomen, bone scans and magnetic resonance images of the brain prior to chemotherapy and at least every 6 weeks during chemotherapy. Tumor response was evaluated following chemotherapy according to the RECIST (Response Evaluation Criteria for Solid Tumors).

Diagnosis of IIP and AE. IIP patients were diagnosed histologically when they showed usual interstitial pneumonia (UIP) or non-specific interstitial pneumonia (NSIP) by surgical lung biopsy. However, without histological evidence, they were diagnosed as having clinical idiopathic pulmonary fibrosis (IPF), a type of IIP, on the basis of high-resolution computed tomography (HRCT) scans of chest and/or clinical findings, including basal predominant subpleural reticular abnormality with traction bronchiectasis and honeycomb cysts and without atypical features of IPF, auscultation of fine crackles, presence of clubbed fingers, results of pulmonary function tests and results of blood examinations [i.e., lactate dehydrogenase (LDH) and KL-6 levels]. Other diseases, including connective tissue disease, infection and hypersensitivity pneumonia, were excluded. The patients had been clinically stable with no disease exacerbation for at least three months prior to diagnosis. All the patients were diagnosed as having IIP by at least three respirologists (M.O., M.T. and K.F.) in accordance with the clinical criteria established by the American Thoracic Society (ATS)/European Respiratory Society (ERS), as reported previously (3).

A diagnosis of AE in IIP patients was made in accordance with the criteria detailed in previous studies $(8,9)$, as follows: i) previous or concurrent diagnosis of IIP; ii) worsening of dyspnea within days to weeks (generally <30 days); iii) evidence of abnormal gas exchange as defined by a low partial pressure of arterial oxygen $\left(\mathrm{PaO}_{2}\right) /$ percentage of inspired oxygen $\left(\mathrm{FiO}_{2}\right)$ ratio or a decrease in $\mathrm{PaO}_{2}$; iv) new radiographic opacities with new bilateral ground-glass abnormality and/or consolidation superimposed on a background reticular or honeycomb pattern consistent with IIP; and v) an absence of an alternative explanation, such as pulmonary infection, left heart failure, pulmonary embolism or an identifiable cause of acute lung injury.

Statistical methods. All values are presented as mean \pm SD. The Fisher's exact and Wilcoxon tests were used to analyze the significance of the associations between NSCLC-IIP with $\mathrm{AE}$ and without $\mathrm{AE}$ and other patient characteristics. Progression-free survival (PFS) was defined as the time between the start of chemotherapy and the date when disease progression began. Patients without progression were regarded as censored at the date of the last follow-up. Overall survival (OS) was defined as the time between the onset of chemotherapy and the date of mortality due to any cause. Patients were regarded as censored if they were alive on the date of the last follow-up. Curves for PFS and OS were estimated by the Kaplan-Meier method, and the differences in survival functions were compared using the log-rank test. The Cox proportional hazards model was applied to examine the prognostic factors significantly associated with PFS or OS after adjustment for other factors. All tests were two-sided, and $\mathrm{P}<0.05$ was considered to indicate a statistically significant difference. All the statistical analyses were conducted using JMP version 8 software (SAS Institute Inc., Cary, NC, USA).

\section{Results}

Characteristics of NSCLC-IIP patients receiving chemotherapy. Table I shows the characteristics of the 22 patients with NSCLC-IIP. A full explanation regarding the potential risks and benefits was provided to all the patients with NSCLC with IIP. As a result, 22 patients received 1 to 4 cycles of chemotherapy (median, 3 cycles). The treatment regimens consisted of CBDCA and TXL ( $\mathrm{n}=19)$, cisplatin-vinorelbine $(n=2)$ and cisplatin-docetaxel $(n=1)$. Tumor response, evalu- 
Table I. Patient characteristics.

\begin{tabular}{|c|c|c|}
\hline Characteristics & $\begin{array}{l}\text { NSCLC with IIP } \\
\text { chemotherapy }\end{array}$ & NSCLC \\
\hline Age (years), median (range) & $70(40-76)$ & $66(35-84)$ \\
\hline \multicolumn{3}{|l|}{ Gender, $\mathrm{n}$} \\
\hline Male & 21 & 172 \\
\hline Female & 1 & 104 \\
\hline \multicolumn{3}{|l|}{ Histology, n } \\
\hline Adenocarcinoma & 11 & 203 \\
\hline Squamous cell carcinoma & 7 & 57 \\
\hline Adenosquamous cell carcinoma & 0 & 3 \\
\hline Large cell carcinoma & 2 & 9 \\
\hline Non-small cell carcinoma & 2 & 4 \\
\hline \multicolumn{3}{|l|}{ Smoking status, $\mathrm{n}$} \\
\hline Never & 0 & 105 \\
\hline Smoker & 22 & 171 \\
\hline \multicolumn{3}{|l|}{ Performance status, $\mathrm{n}$} \\
\hline 0 & 12 & 186 \\
\hline 1 & 10 & 60 \\
\hline 2 & 0 & 30 \\
\hline \multicolumn{3}{|l|}{ Stage, $\mathrm{n}$} \\
\hline IIIA & 1 & 10 \\
\hline IIIB & 6 & 28 \\
\hline IV or recurrent & 15 & 238 \\
\hline \multicolumn{3}{|l|}{ Regimen, $\mathrm{n}$} \\
\hline CDDP-based chemotherapy & 3 & 66 \\
\hline CBDCA-based chemotherapy & 19 & 192 \\
\hline Others (non-platinum) & 0 & 18 \\
\hline Cycle, median (range) & $3(1-4)$ & $3(1-6)$ \\
\hline
\end{tabular}

NSCLC, non-small cell lung cancer; IIP, idiopathic interstitial pneumonia. CDDP, cisplatin; CBDCA, carboplatin.

ated by RECIST, was partial response (PR) in 8 patients, stable disease (SD) in 9 and progressive disease (PD) in 5. The response rate (PR and $\mathrm{SD}$ ) was $72.3 \%$. At the time of analysis, the median follow-up time for NSCLC-IIP patients who had received chemotherapy was 163 days (range, 46-589).

Poorer prognosis in NSCLC-IIP patients compared with NSCLC patients without IIP following chemotherapy. To clarify the consequences of concomitant IIP in NSCLC patients receiving chemotherapy, we compared 22 NSCLC-IIP patients with 276 NSCLC patients without IIP (172 males, 104 females) who had received chemotherapy. As shown in Table I, NSCLC patients without IIP received 1 to 6 cycles of chemotherapy (median, 3 cycles) comprising CBDCA-based treatment $(n=192)$, cisplatin-based treatment $(n=66)$ and non-platinum regimens $(n=18)$. Tumor response, evaluated by RECIST, was complete response (CR) in 6 patients, $\mathrm{PR}$ in $90, \mathrm{SD}$ in 95 and PD in 85 . The response rate (PR and SD) was $69.2 \%$. At the time of analysis, the median follow-up times for NSCLC patients without and with IIP were 400 (range, 14-3,424) and
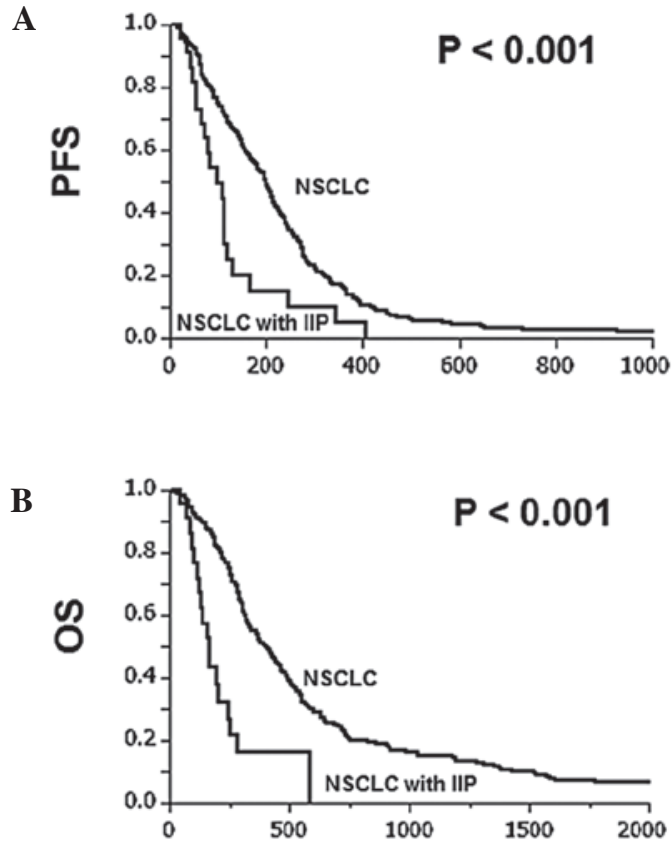

Time (days)

Figure 1. Kaplan-Meier survival curves of (A) PFS and (B) OS in NSCLC patients with or without IIP, who received chemotherapy alone. PFS, progression-free survival; OS, overall survival; NSCLC, non-small cell lung cancer; IIP, idiopathic interstitial pneumonia.

163 days (range, 46-589), respectively. Univariate Cox analysis was carried out to identify the factors that were significantly associated with PFS and OS in all the NSCLC patients receiving chemotherapy, including those with and without IIP (Table II). Poor PS $(\mathrm{P}=0.004)$ and concurrent IIP $(\mathrm{P}<0.001)$ were negative predictors of PFS. For OS, age $(\mathrm{P}=0.028)$, gender $(\mathrm{P}<0.001)$, smoking $(\mathrm{P}<0.001)$, $\mathrm{PS}(\mathrm{P}<0.001)$ and concurrent IIP $(\mathrm{P}<0.001)$ were prognostic. None of the other factors examined were significantly correlated with PFS or OS. Fig. 1 shows the Kaplan-Meier survival curves for NSCLC patients with and without IIP. NSCLC patients with IIP had a significantly shorter median PFS (95.0 vs. 199.5 days, $\mathrm{P}<0.001$ ) and OS (163.0 vs. 400.0 days, $\mathrm{P}<0.001)$ than those without IIP. In addition, the factors that were significantly associated with PFS or OS in NSCLC patients were evaluated by applying Cox regression models adjusting for possible confounding factors. The factors that were of potentially prognostic significance in the univariate analysis were entered into the Cox proportional hazards model: performance status and concomitant IIP for PFS; age, gender, smoking, performance status and concurrent IIP for OS. Table III shows the correlation between incidence of AE and various clinical characteristics, including gender, histology, smoking status, performance status, stage, treatment modality, LDH, KL-6 and \%VC. However, none of the other factors were associated with incidence of $\mathrm{AE}$.

Risk of AE in NSCLC-IIP patients. The incidence of AE was $13.6 \%(3 / 22)$ among NSCLC-IIP patients who received chemotherapy. Table IV shows the clinical characteristics of the NSCLC-IIP patients who developed AE following chemotherapy. All the patients with AE had been treated with the CBDCA and TXL combination. 
$\stackrel{\infty}{\stackrel{2}{0}}$

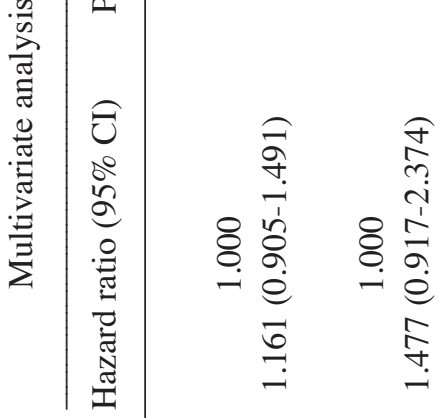

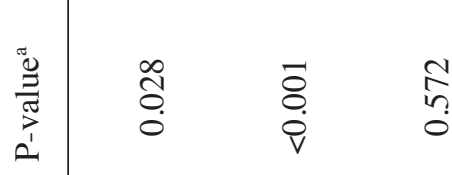

2

焉

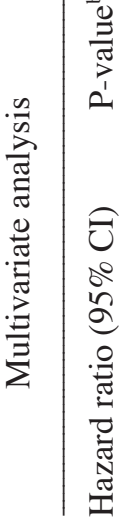

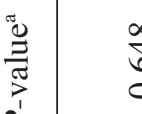

$\stackrel{\infty}{0}$

$\stackrel{0}{n}$

กิ

?ñ

$\stackrel{0}{8} \quad \stackrel{m}{0}$

ริ

\&.

8

空 氶

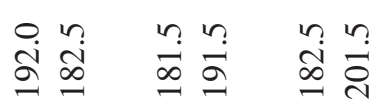

$\stackrel{n}{\infty}$

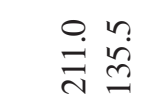

กิ

กุ

กั

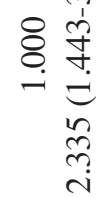

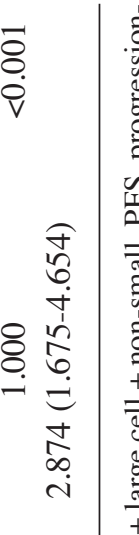

$\overrightarrow{8}$

ํㅜㅇ

के

$=$

호

$\stackrel{2}{2}$

$\stackrel{\nabla}{\sim}$

$\stackrel{2}{2}$

$\approx 8$

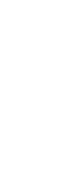

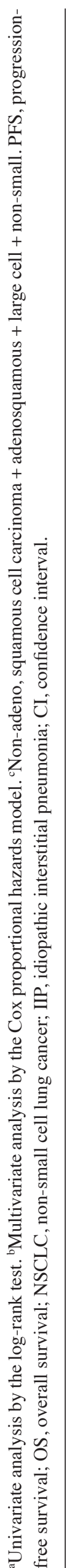


Table III. Factors associated with AE in NSCLC complicated by IIP.

\begin{tabular}{|c|c|c|c|}
\hline \multirow[b]{2}{*}{ Characteristics } & \multicolumn{2}{|c|}{ NSCLC patients with IIP } & \multirow[b]{2}{*}{ P-value } \\
\hline & $\mathrm{AE}(-) \mathrm{n}=19$ & $\mathrm{AE}(+) \mathrm{n}=3$ & \\
\hline Age (years), median (range) & $70(45-76)$ & $66(66-75)$ & \\
\hline \multicolumn{4}{|l|}{ Gender, $\mathrm{n}$} \\
\hline Male & 18 & 3 & \multirow[t]{2}{*}{$1.000^{\mathrm{a}}$} \\
\hline Female & 1 & 0 & \\
\hline \multicolumn{4}{|l|}{ Histology, n } \\
\hline Adenocarcinoma & 9 & 2 & \multirow[t]{2}{*}{$1.000^{\mathrm{a}}$} \\
\hline Non-adenocarcinoma & 10 & 1 & \\
\hline \multicolumn{4}{|l|}{ Smoking status, $\mathrm{n}$} \\
\hline Never & 1 & 0 & \multirow[t]{2}{*}{$1.000^{\mathrm{a}}$} \\
\hline Smoker & 18 & 3 & \\
\hline \multicolumn{4}{|l|}{ Perfomance status, $\mathrm{n}$} \\
\hline 0 & 12 & 0 & \multirow[t]{2}{*}{$0.779^{\mathrm{a}}$} \\
\hline 1 & 7 & 3 & \\
\hline \multicolumn{4}{|l|}{ Stage, $n$} \\
\hline III & 7 & 0 & \multirow[t]{2}{*}{$0.5227^{\mathrm{a}}$} \\
\hline IV or recurrent & 12 & 3 & \\
\hline \multicolumn{4}{|l|}{ Regimen, $\mathrm{n}$} \\
\hline CDDP-based chemotherapy & 3 & 0 & \multirow[t]{2}{*}{$1.000^{\mathrm{a}}$} \\
\hline CBDCA-based chemotherapy & 16 & 3 & \\
\hline $\mathrm{LDH}$, mean $\pm \mathrm{SD}$ & $280.6 \pm 105.6$ & $374 \pm 308.8$ & $0.9238^{\mathrm{b}}$ \\
\hline KL-6, mean \pm SD & $1373.8 \pm 1073.3$ & $950 \pm 298.3$ & $0.6323^{\mathrm{b}}$ \\
\hline$\% \mathrm{VC}$, mean $\pm \mathrm{SD}$ & $89 \pm 18.9$ & $76.1 \pm 21.4$ & $0.3379^{\mathrm{b}}$ \\
\hline
\end{tabular}

${ }^{a}$ By Fisher's exact test; ${ }^{b}$ By Wilcoxon test. NSCLC, non-small cell lung cancer; IIP, idiopathic interstitial pneumonia; LDH, lactate dehydrogenase; $\mathrm{AE}$, acute exacerbation; CDDP, cisplatin; CBDCA, carboplatin.

Table IV. Characteristics of the three patients with AE.

\begin{tabular}{|c|c|c|c|c|c|c|c|c|c|c|}
\hline No. & $\begin{array}{l}\text { Age } \\
\text { (years) }\end{array}$ & Gender & PS & $\begin{array}{c}\text { Smoking } \\
\text { (packs/year) }\end{array}$ & Histology & Stage & Regimen & $\begin{array}{c}\text { Clinical } \\
\text { symptoms }\end{array}$ & $\begin{array}{l}\text { Onset (days from } \\
\text { chemotherapy) }\end{array}$ & $\begin{array}{l}\text { Survival } \\
\text { (days) }\end{array}$ \\
\hline 1 & 73 & M & 1 & 80 & Non-small & IV & CBDCA + PTX & Fever & 132 & 169 \\
\hline 2 & 67 & $\mathrm{M}$ & 1 & 100 & Adenocarcinoma & IV & CBDCA + PTX & Dyspnea & 53 & 138 \\
\hline 3 & 66 & M & 1 & 40 & Adenocarcinoma & IV & CBDCA + PTX & Dyspnea & 52 & 130 \\
\hline
\end{tabular}

CBDCA, carboplatin; PTX, paclitaxel; M, male; PS, performance status; AE, acute exacerbation.

\section{Discussion}

Patients with LC are known to be frequently complicated by IIP. However, there has been little information regarding the optimal treatment approach for advanced NSCLC-IIP. In the present study, to investigate the feasibility and efficacy of chemotherapy for NSCLC-IIP patients, we retrospectively examined 22 NSCLC-IIP patients who received chemotherapy. Consistent with our findings, Minegishi et al also recently reported the efficacy of chemotherapy with CBDCA and TXL in 18 NSCLC-IIP patients, who showed a response rate of $61 \%$, a median PFS of 5.3 months and a median OS of 10.6 months (10). Based on these findings, chemotherapy may be recommended as a feasible option for NSCLC-IIP patients, if the risks of adverse effects are acceptable.

It has been speculated that NSCLC-IIP patients who receive chemotherapy would have a higher risk of $\mathrm{AE}$, which is the most serious adverse event associated with IIP. However, there has been little information concerning AE of IIP following chemotherapy. AE is a well-known phenomenon that develops during the natural course of IIP in $14-21 \%$ of affected patients. Kim et al demonstrated that the incidence 
rate of $\mathrm{AE}$ in IPF patients was $8.5 \%$ within 1 year of diagnosis and $9.6 \%$ within 2 years (11). In the present study, $13.6 \%$ of IIP patients with NSCLC (3 out of 22) developed AE. Similarly, Minegishi et al demonstrated that the incidence rate of $\mathrm{AE}$ was 5.6 and $18 \%$ following the first- and second-line chemotherapy with CBDCA and TXL, respectively (10). Kenmotsu et al also reported that the incidence rate of $\mathrm{AE}$ was $13 \%$ in CBDCA and $1 \%$ in TXL (12). Taken together, these results suggest that chemotherapy, particularly that with the combination of CBDCA and TXL, may be of acceptable toxicity and feasible for patients with NSCLC-IIP who have good performance status.

Since the clinical outcomes of NSCLC-IIP have not been well studied, it has been controversial whether concurrent IIP would affect the prognosis of NSCLC. It has been previously reported that the outcome of LC patients with IIP was worse than that of patients without IIP (13). By contrast, another study has shown that the survival of patients with IIP and LC did not differ significantly from that of patients with IIP or LC alone (7). Since there has been no clear conclusion, we examined the effect of concurrent IIP in the outcomes of NSCLC patients in the present study. Multivariate analysis demonstrated that in NSCLC patients receiving chemotherapy, IIP was a significantly unfavorable factor for PFS and OS. Nevertheless, considering that the response rates to chemotherapy were similar between NSCLC patients with and without IIP (72.3 vs. $69.2 \%$ ), the poor prognosis of NSCLC-IIP patients may, at least in part, be due to the natural course of IIP, rather than to poorer response to treatments.

In summary, the present findings suggest that chemotherapy is a feasible option for NSCLC-IIP. Nevertheless, it should be noted that there are some limitations in the present study. First, the number of NSCLC-IIP patients was relatively small, and the population was heterogeneous. Second, the retrospective nature did not allow for a standardized measure of PFS. Therefore, a larger-scale prospective randomized control study employing homogeneous standard regimens is required in order to evaluate more precisely the feasibility and efficacy of chemotherapy for patients with NSCLC-IIP.

\section{Acknowledgements}

We thank Hisamichi Aizawa for useful advice.

\section{References}

1. Parkin DM, Bray FI and Devesa SS: Cancer burden in the year 2000: the global picture. Eur J Cancer 37 (Suppl 8): S4-S66, 2001.

2. Turner-Warwick M, Lebowits M, Burrows B and Johnson A: Cryptogenic fibrosing alveolitis and lung cancer. Thorax 35: 496-499, 1980

3. American Thoracic Society/European Respiratory Society: American Thoracic Society/European Respiratory Society international multidisciplinary consensus classification of the idiopathic interstitial pneumonias. This joint statement of the American Thoracic Society (ATS), and the European Respiratory Society (ERS) was adopted by the ATS board of directors, June 2001 and by the ERS Executive Committee, June 2001. Am J Respir Crit Care Med 165: 277-304, 2002.

4. Katzenstein AL and Myers JL: Idiopathic pulmonary fibrosis. clinical relevance of pathologic classification. Am J Respir Crit Care Med 157: 1301-1315, 1998.

5. Kawasaki H, Nagai K, Yokose T, et al: Clinicopathological characteristics of surgically resected lung cancer associated with idiopathic pulmonary fibrosis. J Surg Oncol 76: 53-57, 2001.

6. Park J, Kim DS, Shim TS, et al: Lung cancer in patients with idiopathic pulmonary fibrosis. Eur Respir J 17: 1216-1219, 2001.

7. Aubry MC, Myers JL, Douglas WW, et al: Primary pulmonary carcinoma in patients with idiopathic pulmonary fibrosis. Mayo Clin Proc 77: 763-770, 2002.

8. Collard HR, Moore BB, Flaherty KR, et al: Acute exacerbations of idiopathic pulmonary fibrosis. Am J Respir Crit Care Med 176: 636-643, 2007.

9. Agarwal R and Jindal SK: Acute exacerbation of idiopathic pulmonary fibrosis: a systematic review. Eur J Intern Med 19: 227-235, 2008

10. Minegishi Y, Sudoh J, Kuribayashi H, et al: The safety and efficacy of weekly paclitaxel in combination with carboplatin for advanced non-small cell lung cancer with idiopathic interstitial pneumonias. Lung Cancer 71: 70-74, 2011.

11. Kim DS, Park JH, Park BK, Lee JS, Nicholson AG and Colby T: Acute exacerbation of idiopathic pulmonary fibrosis: frequency and clinical features. Eur Respir J 27: 143-150, 2006.

12. Kenmotsu H, Naito T, Kimura M, et al: The risk of cytotoxic chemotherapy-related exacerbation of interstitial lung disease with lung cancer. J Thorac Oncol 6: 1242-1246, 2011.

13. Miyazaki K, Kurishima K, Kagohashi K, Kawaguchi M, Ishikawa H, Satoh H and Hizawa N: Serum KL-6 levels in lung cancer patients with or without interstitial lung disease. J Clin Lab Anal 24: 295-299, 2010. 